\title{
New neon-abundance results in Galactic WN and WC stars
}

\author{
John-David T. Smith \\ Steward Observatory, University of Arizona, \\ 933 North Cherry Avenue, Tucson, AZ 85721, USA
}

\section{Introduction}

The fast, dense winds which characterize Wolf-Rayet stars obscure their underlying cores, and complicate the verification of evolving core and nucleosynthesis models. A powerful technique for probing WR core evolution involves measuring abundances of wind-borne nuclear processed elements. Neon, in particular, undergoes a remarkable change in abundance during the later stages of a WR star's lifetime. By the end of the WC phase, it becomes the fourth most abundant element, after $\mathrm{He}, \mathrm{C}$ and $\mathrm{O}$ (Maeder 1983).

Early low-resolution neon abundance measurements by Aitken et al. (1982) of the brightest nearby WR star, $\gamma^{2}$ Velorum (WR 11, WC8+O7.5III-V) were confirmed by van der Hucht \& Olnon (1985) using IRAS spectra, and both results agreed very well with the WR core evolution models, which predict $\mathrm{Ne} / \mathrm{He} \simeq 12 \times$ the cosmic value of $5.5 \times 10^{-4}$. Barlow et al. (1988) revised the abundance downwards, putting it quite close to cosmic levels. This serious discrepancy persisted until recently, when Dessart et al. (2000) used updated distance and mass-loss rates and ISO data to bring the Ne abundance again within $30 \%$ of the predicted value.

\section{Observations and results}

The 8-13 $\mu \mathrm{m}$ spectra were obtained April-July 1998, as part of a larger midinfrared spectral survey of northern Galactic WR stars (Smith \& Houck 2001) with SCORE, the SIRTF Cornell Echelle Spectrograph, on the Palomar 5m telescope. Two of the neon spectra are shown in Figure 1.

The fractional abundance $\gamma_{\mathrm{Ne}^{+}}$by number was calculated from the measured line parameters, using a two-level approximation for the $\mathrm{Ne}^{+}$ground state, with chemical composition, distance, mass loss rate, and terminal velocity taken from the literature and analogy with survey stars of the same type. The resulting abundance values with respect to helium are listed in Table 1.

The WC9 star WR 121 shows neon abundance $\sim 7.5 \times$ the cosmic value. (The higher ionization state $\mathrm{Ne}^{++}$can be traced by [S IV] $10.5 \mu m$, which is absent in all spectra except WR 105, indicating $\mathrm{Ne}^{+}$accounts for nearly all the neon in these stars' winds.) The three WN stars show near cosmic abundance levels, as expected for cores which have yet to undergo advanced nuclear processing. In WR 105 , the $\mathrm{Ne}^{+} / \mathrm{S}^{3+}$ abundance ratio, which is immune to errors in mass 


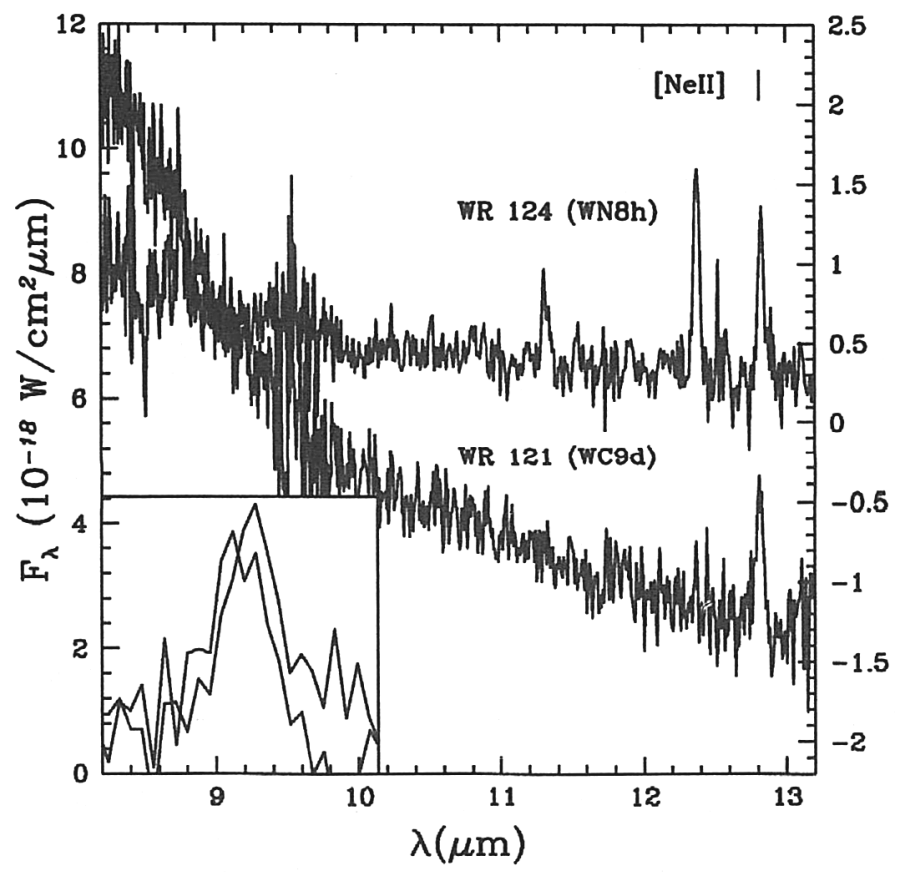

Figure 1. Two of the four stars with neon; [Ne II] line profiles inset.

Table 1. Neon abundances in WR stars

\begin{tabular}{ccc}
\hline \hline WR & type & $\mathrm{Ne}^{+} / \mathrm{He}\left(\times 10^{-4}\right)$ \\
\hline 105 & WN9h & 8.8 \\
116 & WN8h & 5.3 \\
121 & WC9d & 41. \\
124 & WN8h & 4.2 \\
\hline \hline
\end{tabular}

loss rate, distance, etc., is consistent with the solar $\mathrm{Ne} / \mathrm{S}$ value, within the ionization distribution uncertainties.

\section{References}

Aitken, D.K., Roche, P.F., Allen, D.A. 1982, MNRAS (Letters) 200, 69P

Barlow, M.J., Roche, P.F., Aitken, D.K. 1988, MNRAS 232, 821

Dessart, L., Crowther, P.A., Hillier, D.J., Willis, A.J., Morris, P.W., van der Hucht, K.A. 2000, MNRAS 315, 407

van der Hucht, K.A., Olnon, F.M. 1985, A\&A (Letters) 149, L17

Maeder, A. 1983, A\&A 120, 113

Smith, J.D.T., Houck, J.R. 2001, AJ 121, 2115 